\title{
Estimation for the Triangular Distribution under Progressive Type-II Censoring
}

\author{
Suk-Bok Kang'1), Jun-Tae Han²), Won-Tae Jung ${ }^{3)}$
}

\begin{abstract}
In this paper, we derive the approximate maximum likelihood estimators(AMLEs) and maximum likelihood estimator of the scale parameter in a triangular distribution based on progressive Type-II censored samples. We compare the proposed estimators in the sense of the mean squared error through Monte Carlo simulation for various progressive censoring schemes.
\end{abstract}

Keywords: Approximate maximum likelihood estimator; progressive Type-II censoring; triangular distribution.

\section{Introduction}

The cumulative distribution function(cdf) and the probability density function(pdf) of the random variable having the triangular distribution are given by

$$
F(x)= \begin{cases}\frac{x^{2}}{\lambda \sigma^{2}}, & 0 \leq x<\lambda \sigma, \\ 1-\frac{1}{(1-\lambda)}\left(1-\frac{x}{\sigma}\right)^{2}, & \lambda \sigma \leq x \leq \sigma\end{cases}
$$

and

$$
f(x)= \begin{cases}\frac{2 x}{\lambda \sigma^{2}}, & 0 \leq x<\lambda \sigma, \\ \frac{2(\sigma-x)}{(1-\lambda) \sigma^{2}}, & \lambda \sigma \leq x \leq \sigma,\end{cases}
$$

where $\sigma>0$ and $0<\lambda<1$.

A triangular distribution is specified by its minimum, maximum and mean values. It doses not have to be symmetric, it can be skewed to the left or right by entering a mean value less than or greater than the average of the minimum and maximum values.

The distribution emerges in numerous papers dealing with the Project Evaluation and Review Technique-PERT (see, Moder and Rodgers, 1962; Keefer and Verdini, 1993; Johnson, 1997).

As a special case, when $\sigma=1$ and $\lambda=1 / 2$, it become symmetric standard triangular distribution. The mode of this triangular distribution with $\sigma=1$ is $x=\lambda$ and the value of the $p d f$ at the mode is always 2, whatever the value of $\lambda$ (see Figure 1.1).

1) Professor, Department of Statistics, Yeungnam University, Gyeongsan 712-749, Korea.

2) Researcher, Institute for National Health Insurance, National Health Insurance Corporation, Seoul 121-749, Korea. Correspondence: maru@nhic.or.kr

3) Graduate student, Department of Statistics, Yeungnam University, Gyeongsan 712-749, Korea. 


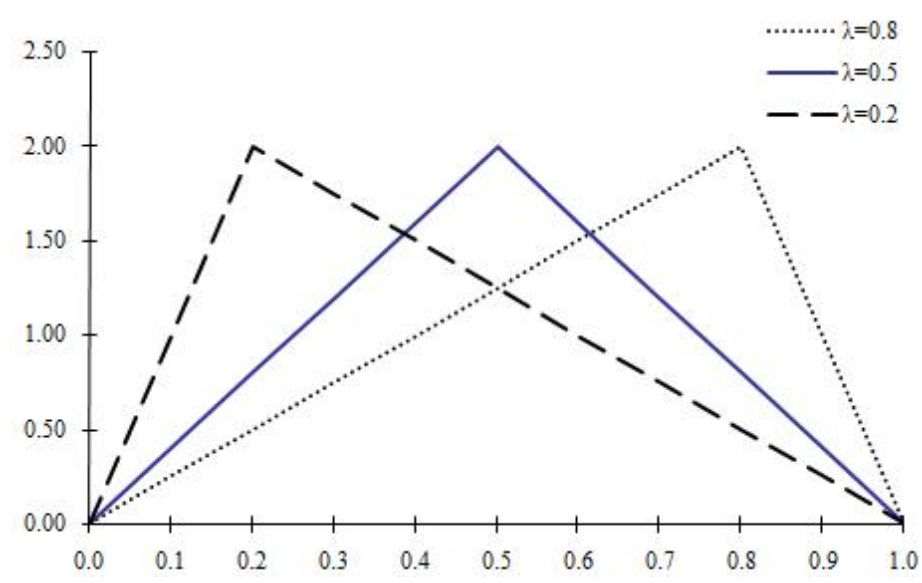

Figure 1.1: Showing the $p d f$ of triangular distribution with $\sigma=1$ and $\lambda=0.2,0.5,0.8$.

Johnson (1997) studied the possibility of using the more intuitively obvious triangular distribution as a proxy for the beta distribution. von Drop and Kotz (2002) investigated the four-parameter two-sided power(TSP) distribution which is an extension of the threeparameter triangular distribution. Some properties of the triangular distribution was studied by Balakrishnan and Nevzorov (2003).

In most cases of censored and truncated samples, the maximum likelihood method does not provide explicit estimators. So we need another method for the purpose of providing the explicit estimators. The approximated maximum likelihood estimation method was first developed by Balakrishnan (1989) for the purpose of providing the explicit estimators of the scale parameter in the Rayleigh distribution. Balakrishnan and Wong (1991) derived the approximated maximum likelihood estimator(AMLE) of the scale parameter of the half-logistic distribution with Type-II right censoring. They also studied the bias and variance of proposed estimator. Kang (1996) obtained the AMLE for the scale parameter of the double exponential distribution based on Type-II censored samples and showed that the proposed estimator is generally more efficient than the best linear unbiased estimator(BLUE) and the optimum unbiased absolute estimator.

The scheme of the progressive Type-II censoring has been suggested in the field of life-testing experiments. It makes allowance for the removal of live units from the experiment at various stages in an attractive feature as it will potentially save a lot for the experimenter in terms of cost and time.

Viveros and Balakrishnan (1994) derived explicit expressions for the BLUEs of the parameters of the exponential distributions. They also developed a conditional method of inference to derive exact confidence intervals for the parameters of the extreme value distribution. Balasooriya et al. (2000) studied the progressively censored sampling plan for the Weibull distribution.

$\mathrm{Ng}$ et al. (2002) discussed the estimation of parameters from progressively censored data using the EM algorithm. Balakrishnan et al. (2003) suggested point and interval estimation for Gaussian distribution based on progressive Type-II censored samples. 
Balakrishnan et al. (2004) studied point and interval estimation for the extreme value distribution based on progressively Type-II censored samples. Balakrishnan and Asgharzadeh (2005) discussed the maximum likelihood estimator(MLE) of the scale parameter of the half-logistic distribution based on progressive Type-II censored samples. They also provided the AMLE of the scale parameter of the half-logistic distribution based on progressive Type-II censored samples. Lin et al. (2006) discussed the MLEs of the parameters of the log-gamma distribution based on progressive Type-II censored samples and they derived the AMLEs of the parameters and used them as initial values in the determination of the MLEs through the Newton-Raphson method. Kim (2006) dealt with the problem of estimating parameters of Burr Type-XII distribution, on the basis of a general progressive Type-II censored samples using Bayesian viewpoints. Seo and Kang (2007) proposed the AMLEs of the scale parameter when the location parameter is known and the AMLE of the location parameter when the scale parameter is known in the two-parameter Rayleigh distribution based on progressive Type-II censored samples. They also proposed the AMLEs of the location and scale parameters in the two-parameter Rayleigh distribution based on progressive Type-II censored samples when two parameters are unknown.

Recently, Lee et al. (2008) proposed the AMLEs of the scale parameter in a triangular distribution based on multiply Type-II censored samples by the approximate maximum likelihood estimation methods. Han and Kang (2008) derived the AMLEs of the scale parameter and the location parameter in a double Rayleigh distribution based on multiply Type-II censored samples.

In this paper, we derive the AMLEs and the MLE of the scale parameter $\sigma$ when the shape parameter $\lambda$ is known in the triangular distribution under progressive TypeII censoring. The scale parameter $\sigma$ is estimated by approximate maximum likelihood estimation method using two different types of Taylor series expansions. We compare the proposed estimators in the sense of the mean squared error through Monte Carlo simulation for various progressive censoring schemes.

\section{Maximum Likelihood Estimation}

We will discuss the maximum likelihood estimation of the scale parameter based on progressive Type-II censored samples. Let $X_{1: m: n}, \ldots, X_{m: m: n}$ be a progressively Type-II censored sample from a triangular distribution, with the progressive censoring scheme $\left(R_{1}, \ldots, R_{m}\right)$.

If $R_{1}=\cdots=R_{m}=0$, we have $n=m$ which corresponds to the complete sample. If $R_{1}=\cdots=R_{m-1}=0$, then $R_{m}=n-m$ which corresponds to the conventional Type-II right censoring sample. This generalized censoring scheme is called Progressively Type-II censoring. Further details and extensive references on progressive censoring may be obtained from the book by Balakrishnan and Aggarwala (2000).

The likelihood function based on the progressively Type-II censored sample is given by

$$
L=C \prod_{i=1}^{m} f\left(x_{i: m: n} ; \sigma, \lambda\right)\left[1-F\left(x_{i: m: n} ; \sigma, \lambda\right)\right]^{R_{i}}
$$

where $C=n\left(n-1-R_{1}\right)\left(n-2-R_{1}-R_{2}\right) \cdots\left(n-m+1-R_{1}-\cdots-R_{m-1}\right)$. 
The random variable $Z_{i: m: n}=X_{i: m: n} / \sigma$ has a standard triangular distribution with $p d f$ and $c d f$ :

$$
f\left(z_{i: m: n}\right)= \begin{cases}\frac{2 z_{i: m: n}}{\lambda}, & 0 \leq z \leq \lambda \\ \frac{2\left(1-z_{i: m: n}\right)}{(1-\lambda)}, & \lambda<z \leq 1\end{cases}
$$

and

$$
F\left(z_{i: m: n}\right)= \begin{cases}\frac{z_{i: m: n}^{2}}{\lambda}, & 0 \leq z \leq \lambda, \\ 1-\frac{\left(1-z_{i: m: n}\right)^{2}}{(1-\lambda)}, & \lambda<z \leq 1 .\end{cases}
$$

From the equation (2.1), the log-likelihood function may be written as

$$
\ln L=K-m \ln \sigma+\sum_{i=1}^{m} \ln f\left(z_{i: m: n}\right)+\sum_{i=1}^{m} R_{i} \ln \left[1-F\left(z_{i: m: n}\right)\right],
$$

where $K$ is a constant.

On differentiating the log-likelihood function with respect to $\sigma$ in turn and the equation to zero, we obtain the estimating equation as

$$
\frac{\partial \ln L}{\partial \sigma}=-\frac{1}{\sigma}\left[m+\sum_{i=1}^{m} \frac{f^{\prime}\left(z_{i: m: n}\right)}{f\left(z_{i: m: n}\right)} z_{i: m: n}-\sum_{i=1}^{m} R_{i} \frac{f\left(z_{i: m: n}\right)}{1-F\left(z_{i: m: n}\right)} z_{i: m: n}\right]=0 .
$$

We can find the MLE of $\sigma$ as values $\hat{\sigma}$ that maximize the log-likelihood function in (2.2) by solving the equation $\partial \ln L / \partial \sigma=0$. Since the equation (2.3) cannot be solved explicitly, a numerical method may be used for the numerical determination of the MLE. We evaluated the mean squared errors of $\sigma$ using the bisection method. These values are given in Table 4.1.

\section{Approximate Maximum Likelihood Estimators}

Since the log-likelihood equation do not admit explicit solutions, it will be desirable to consider an approximation to the likelihood equation which provide us with explicit estimator for the scale parameter.

Let

$$
\xi_{i: m: n}=F^{-1}\left(p_{i: m: n}\right)= \begin{cases}\sqrt{p_{i: m: n} \lambda}, & p_{i: m: n} \leq \lambda \\ 1+\sqrt{\left(1-p_{i: m: n}\right)(1-\lambda)}, & p_{i: m: n}>\lambda\end{cases}
$$

where $q_{i: m: n}=1-p_{i: m: n}$ and

$$
p_{i: m: n}=1-\prod_{j=m-i+1}^{m} \frac{j+R_{m-j+1}+\cdots+R_{m}}{j+1+R_{m-j+1}+\cdots+R_{m}}, \quad i=1, \ldots, m .
$$


First, we can approximate the following functions by Taylor series expansion as

$$
\frac{f^{\prime}\left(z_{i: m: n}\right)}{f\left(z_{i: m: n}\right)} z_{i: m: n} \simeq \alpha_{1 i}+\beta_{1 i} z_{i: m: n}
$$

and

$$
\frac{f\left(z_{i: m: n}\right)}{1-F\left(z_{i: m: n}\right)} z_{i: m: n} \simeq \gamma_{1 i}+\delta_{1 i} z_{i: m: n}
$$

where

$$
\begin{aligned}
& \alpha_{1 i}= \begin{cases}\frac{\xi_{i: m: n}^{2}}{(1-\lambda) q_{i: m: n}}, \quad p_{i: m: n} \geq \lambda, \\
1, \quad p_{i: m: n}<\lambda,\end{cases} \\
& \beta_{1 i}= \begin{cases}-\frac{1}{(1-\lambda) q_{i: m: n}}, \quad p_{i: m: n} \geq \lambda, \\
0, \quad p_{i: m: n}<\lambda,\end{cases} \\
& \gamma_{1 i}= \begin{cases}-\frac{2 \xi_{i: m: n}^{2}}{(1-\lambda) q_{i: m: n}}, \quad p_{i: m: n} \geq \lambda, \\
-\frac{2 p_{i: m: n}}{q_{i: m: n}}\left[1+\frac{2 p_{i: m: n}}{q_{i: m: n}}\right], & p_{i: m: n} \geq \lambda,\end{cases} \\
& \delta_{1 i}= \begin{cases}\frac{2}{(1-\lambda) q_{i: m: n}}, & p_{i: m: n}<\lambda .\end{cases}
\end{aligned}
$$

By substituting the equations (3.1) and (3.2) into the equation (2.3), we may approximate the likelihood equation in $(2.3)$ by

$$
\frac{\partial \ln L}{\partial \sigma} \simeq-\frac{1}{\sigma}\left[m+\sum_{i=1}^{m}\left(\alpha_{1 i}+\beta_{1 i} z_{i: m: n}\right)-\sum_{i=1}^{m} R_{i}\left(\gamma_{1 i}+\delta_{1 i} z_{i: m: n}\right)\right]=0
$$

We can derive an estimator of $\sigma$ as follows:

$$
\tilde{\sigma}_{1}=\frac{\sum_{i=1}^{m}\left(R_{i} \delta_{1 i} X_{i: m: n}-\beta_{1 i} X_{i: m: n}\right)}{m+\sum_{i=1}^{m}\left(\alpha_{1 i}-R_{i} \gamma_{1 i}\right)} .
$$

Since $\alpha_{1 i}>0, \gamma_{1 i}<0, \delta_{1 i}>0$ and $\beta_{1 i} \leq 0$, the estimator $\tilde{\sigma}_{1}$ is always positive.

Second, we can approximate the following functions

$$
\frac{f^{\prime}\left(z_{i: m: n}\right)}{f\left(z_{i: m: n}\right)} \simeq \alpha_{2 i}+\beta_{2 i} z_{i: m: n}
$$


and

$$
\frac{f\left(z_{i: m: n}\right)}{1-F\left(z_{i: m: n}\right)} \simeq \gamma_{2 i}+\delta_{2 i} z_{i: m: n}
$$

where

$$
\begin{gathered}
\alpha_{2 i}= \begin{cases}-\frac{1-2 \xi_{i: m: n}}{(1-\lambda) q_{i: m: n}}, & p_{i: m: n} \geq \lambda, \\
\frac{2}{\xi_{i: m: n}}, & p_{i: m: n}<\lambda,\end{cases} \\
\beta_{2 i}= \begin{cases}-\frac{1}{(1-\lambda) q_{i: m: n}}, & p_{i: m: n} \geq \lambda, \\
-\frac{1}{\lambda p_{i: m: n}}, & p_{i: m: n}<\lambda,\end{cases} \\
\gamma_{2 i}= \begin{cases}\frac{2\left(1-2 \xi_{i: m: n}\right)}{(1-\lambda) q_{i: m: n}}, & p_{i: m: n} \geq \lambda, \\
-\frac{4 p_{i: m: n}}{\lambda q_{i: m: n}^{2}} \xi_{i: m: n}, & p_{i: m: n}<\lambda,\end{cases} \\
\delta_{2 i}= \begin{cases}\frac{2}{(1-\lambda) q_{i: m: n}}, & p_{i: m: n} \geq \lambda, \\
\frac{2}{\lambda q_{i: m: n}}\left[1+\frac{2 p_{i: m: n}}{q_{i: m: n}}\right], & p_{i: m: n}<\lambda .\end{cases}
\end{gathered}
$$

By substituting the equations (3.5) and (3.6) into the equation (2.3), we may approximate the likelihood equation in (2.3) by

$$
\frac{\partial \ln L}{\partial \sigma} \simeq-\frac{1}{\sigma}\left[m+\sum_{i=1}^{m}\left(\alpha_{2 i}+\beta_{2 i} z_{i: m: n}\right) z_{i: m: n}-\sum_{i=1}^{m} R_{i}\left(\gamma_{2 i}+\delta_{2 i} z_{i: m: n}\right) z_{i: m: n}\right]=0
$$

Upon solving (3.7) for $\sigma$, we can derive the other estimator of $\sigma$ as follows:

$$
\tilde{\sigma}_{2}=\frac{-A+\sqrt{A^{2}-4 m B}}{2 m},
$$

where

$$
\begin{aligned}
& A=\sum_{i=1}^{m}\left(\alpha_{2 i} X_{i: m: n}-R_{i} \gamma_{2 i} X_{i: m: n}\right), \\
& B=\sum_{i=1}^{m}\left(\beta_{2 i} X_{i: m: n}^{2}-R_{i} \delta_{2 i} X_{i: m: n}^{2}\right) .
\end{aligned}
$$

Upon solving (3.7) for $\sigma$ we get quadratic equation in $\sigma$ which has two roots; however, one of them drops out since $\beta_{2 i}<0$ and $\delta_{2 i}>0$ hence $B<0$. 


\section{The Simulated Results}

The simulations were carried out for sample size $n=10(5) 20,30,40$ and different choices of the effective sample size $m$ and different progressive censoring schemes with the complete sample in each case. For simplicity in notation, we will denote the schemes $(0,0, \ldots, n-m)$ by $((n-m) * 0, n-m)$, for example, $(10 * 0)$ denotes the progressive censoring scheme $(0,0, \ldots, 0)$ and $(3 * 0,2,2,0)$ denotes the progressive censoring scheme $(0,0,0,2,2,0)$.

The mean squared errors of the proposed AMLEs and MLE are simulated by Monte Carlo method(based on 10,000 Monte Carlo runs) for sample size $n=10(5) 20,30,40$ and various choices of censoring under progressive Type-II censored sample when $\lambda=$ $0.2,0.5,0.8$. These values are given in Table 4.1 .

From Table 4.1 , the estimators $\tilde{\sigma}_{1}$ and $\tilde{\sigma}_{2}$ are generally more efficient than the maximum likelihood estimator $\hat{\sigma}$.

The estimator $\tilde{\sigma}_{1}$ is the linear function of available order statistics. So the estimator $\tilde{\sigma}_{1}$ is a more simple estimator than the estimator $\tilde{\sigma}_{2}$. But the estimator $\tilde{\sigma}_{2}$ is generally more efficient than the estimators $\tilde{\sigma}_{1}$ and $\hat{\sigma}$ in the sense of the MSE.

For sample size $n=10$ and the progressive censoring scheme $(5 * 0,4)$ (right TypeII censored sample), the estimators $\tilde{\sigma}_{1}$ and $\tilde{\sigma}_{2}$ are more efficient than the maximum likelihood estimator $\hat{\sigma}$ when $\lambda<0.5$. But the maximum likelihood estimator $\hat{\sigma}$ is more efficient than the estimators $\tilde{\sigma}_{1}$ and $\tilde{\sigma}_{2}$ when $\lambda \geq 0.5$.

The MSE of the estimator $\hat{\sigma}$ decreases as $\lambda$ increases.

As expected, the MSEs of all estimators decrease as sample size $n$ increases.

For fixed sample size, the MSE increases generally as the number of unobserved or missing data $n-m$ increases. The MSE of the estimator $\tilde{\sigma}_{1}$ decreases as $\lambda$ increases when $n \geq 30$.

\section{Discussion}

Application and estimation of the parameters in the triangular distribution was studied by some authors. However, previous studies obtained the maximum likelihood estimator of the shape parameter for complete data.

In this study, we just derive the AMLEs and the MLE of the scale parameter $\sigma$ when the shape parameter $\lambda$ is known in the triangular distribution under progressive TypeII censoring. The scale parameter $\sigma$ is estimated by approximate maximum likelihood estimation method using two different types of Taylor series expansions. We evaluated the mean squared errors of the MLE $\hat{\sigma}$ of $\sigma$ using the bisection method.

However, we can't evaluate the value of the proposed estimators using a raw data because we assume that the shape parameter $\lambda$ is known.

In future study, we will consider the estimation of the scale parameter $\sigma$ and the shape parameter $\lambda$ in the triangular distribution under progressive Type-II censoring when two parameters are unknown and evaluate the value of the proposed estimators using some raw data. 
Table 4.1: The relative mean squared errors for the estimators of the scale parameter $\sigma$

\begin{tabular}{|c|c|c|c|c|c|}
\hline \multicolumn{6}{|c|}{$\lambda=0.2$} \\
\hline$n$ & $m$ & Scheme & $\tilde{\sigma}_{1}$ & $\tilde{\sigma}_{2}$ & $\hat{\sigma}$ \\
\hline \multirow{7}{*}{10} & 10 & $\left(10^{*} 0\right)$ & 0.110442 & 0.044126 & 0.225869 \\
\hline & 6 & $\left(3^{*} 0,2,2,0\right)$ & 0.237637 & 0.114913 & 0.241003 \\
\hline & 6 & $(2 * 0,4,3 * 0)$ & 0.331257 & 0.116440 & 0.203387 \\
\hline & 6 & $(4,5 * 0)$ & 0.150639 & 0.258102 & 0.219007 \\
\hline & 6 & $\left(5^{*} 0,4\right)$ & 0.178378 & 0.107536 & 0.249112 \\
\hline & 5 & $\left(2 * 0,5,2^{*} 0\right)$ & 0.389127 & 0.160948 & 0.211510 \\
\hline & 5 & $(5,4 * 0)$ & 0.165975 & 0.490864 & 0.217374 \\
\hline \multirow{5}{*}{15} & 15 & $\left(15^{*} 0\right)$ & 0.083705 & 0.027246 & 0.226679 \\
\hline & 10 & $(5,9 * 0)$ & 0.111587 & 0.074364 & 0.223873 \\
\hline & 10 & $(4 * 0,3,3 * 0,2,0)$ & 0.199542 & 0.072929 & 0.235607 \\
\hline & 10 & $\left(0,3,6^{*} 0,2,0\right)$ & 0.131942 & 0.117645 & 0.239620 \\
\hline & 10 & $\left(2^{*} 0,1,0,2,0,2,3^{*} 0\right)$ & 0.178967 & 0.096754 & 0.227030 \\
\hline \multirow{5}{*}{20} & 20 & $\left(20^{*} 0\right)$ & 0.067359 & 0.017348 & 0.226764 \\
\hline & 15 & $\left(3^{*} 0,2,4^{*} 0,3,6^{*} 0\right)$ & 0.108554 & 0.070047 & 0.224898 \\
\hline & 10 & $\left(5,2^{*} 0,5,6^{*} 0\right)$ & 0.273374 & 0.075084 & 0.196096 \\
\hline & 10 & $\left(2^{*} 0,1,0,2,0,2,2^{*} 0,5\right)$ & 0.199431 & 0.097799 & 0.248643 \\
\hline & 10 & $\left(2^{*} 0,3,0,2,0,2,2^{*} 0,3\right)$ & 0.185004 & 0.135661 & 0.244515 \\
\hline \multirow{6}{*}{30} & 30 & $\left(30^{*} 0\right)$ & 0.048147 & 0.008315 & 0.227263 \\
\hline & 20 & $(3 * 0,5,3 * 0,5,12 * 0)$ & 0.130541 & 0.049663 & 0.193376 \\
\hline & 20 & $\left(2 * 0,10,17^{*} 0\right)$ & 0.064029 & 0.046222 & 0.223301 \\
\hline & 20 & $(9 * 0,10,10 * 0)$ & 0.175434 & 0.046514 & 0.196909 \\
\hline & 15 & $\left(5,6^{*} 0,10,7^{*} 0\right)$ & 0.231636 & 0.068110 & 0.197318 \\
\hline & 15 & $\left(10,6^{*} 0,5,7^{*} 0\right)$ & 0.161388 & 0.047668 & 0.209251 \\
\hline \multirow{4}{*}{40} & 20 & $\left(8^{*} 0,10,10,10^{*} 0\right)$ & 0.310355 & 0.071330 & 0.174500 \\
\hline & 20 & $\left(2^{*} 0,5,5,5,5,14^{*} 0\right)$ & 0.053318 & 0.189324 & 0.210544 \\
\hline & 20 & $(5,16 * 0,5,5,5)$ & 0.155809 & 0.048901 & 0.249873 \\
\hline & 20 & $\left(3^{*} 0,15,7^{*} 0,5,8^{*} 0\right)$ & 0.120545 & 0.062319 & 0.225352 \\
\hline \multicolumn{6}{|c|}{$\lambda=0.5$} \\
\hline$n$ & $m$ & Scheme & $\tilde{\sigma}_{1}$ & $\tilde{\sigma}_{2}$ & $\hat{\sigma}$ \\
\hline \multirow{7}{*}{10} & 10 & $\left(10^{*} 0\right)$ & 0.115340 & 0.043745 & 0.137189 \\
\hline & 6 & $\left(3^{*} 0,2,2,0\right)$ & 0.237109 & 0.450192 & 0.119298 \\
\hline & 6 & $\left(2 * 0,4,3^{*} 0\right)$ & 0.136415 & 0.089055 & 0.134350 \\
\hline & 6 & $(4,5 * 0)$ & 0.172474 & 0.078036 & 0.140363 \\
\hline & 6 & $\left(5^{*} 0,4\right)$ & 0.359106 & 0.252212 & 0.142993 \\
\hline & 5 & $\left(2 * 0,5,2^{*} 0\right)$ & 0.167735 & 0.136254 & 0.132306 \\
\hline & 5 & $(5,4 * 0)$ & 0.180826 & 0.084186 & 0.141940 \\
\hline \multirow{5}{*}{15} & 15 & $\left(15^{*} 0\right)$ & 0.089663 & 0.031551 & 0.135320 \\
\hline & 10 & $(5,9 * 0)$ & 0.118352 & 0.045633 & 0.138073 \\
\hline & 10 & $(4 * 0,3,3 * 0,2,0)$ & 0.164568 & 0.085849 & 0.146109 \\
\hline & 10 & $\left(0,3,6^{*} 0,2,0\right)$ & 0.173441 & 0.085668 & 0.153623 \\
\hline & 10 & $\left(2^{*} 0,1,0,2,0,2,3^{*} 0\right)$ & 0.063646 & 0.087484 & 0.122142 \\
\hline \multirow{5}{*}{20} & 20 & $\left(20^{*} 0\right)$ & 0.067280 & 0.021811 & 0.136155 \\
\hline & 15 & $\left(3^{*} 0,2,4^{*} 0,3,6^{*} 0\right)$ & 0.058428 & 0.036147 & 0.124082 \\
\hline & 10 & $\left(5,2^{*} 0,5,6^{*} 0\right)$ & 0.107611 & 0.046443 & 0.137877 \\
\hline & 10 & $\left(2^{*} 0,1,0,2,0,2,2^{*} 0,5\right)$ & 0.249820 & 0.165307 & 0.161270 \\
\hline & 10 & $\left(2^{*} 0,3,0,2,0,2,2^{*} 0,3\right)$ & 0.151748 & 0.092580 & 0.177242 \\
\hline
\end{tabular}




\begin{tabular}{|c|c|c|c|c|c|}
\hline \multirow{6}{*}{30} & 30 & $\left(30^{*} 0\right)$ & 0.048006 & 0.013582 & 0.135329 \\
\hline & 20 & $\left(3^{*} 0,5,3^{*} 0,5,12^{*} 0\right)$ & 0.068419 & 0.024527 & 0.136913 \\
\hline & 20 & $(2 * 0,10,17 * 0)$ & 0.064931 & 0.021352 & 0.136673 \\
\hline & 20 & $(9 * 0,10,10 * 0)$ & 0.064245 & 0.027192 & 0.136518 \\
\hline & 15 & $\left(5,6^{*} 0,10,7^{*} 0\right)$ & 0.072519 & 0.035379 & 0.136167 \\
\hline & 15 & $\left(10,6 * 0,5,7^{*} 0\right)$ & 0.074482 & 0.037670 & 0.131893 \\
\hline \multirow{4}{*}{40} & 20 & $\left(8^{*} 0,10,10,10^{*} 0\right)$ & 0.062680 & 0.025358 & 0.138948 \\
\hline & 20 & $\left(2^{*} 0,5,5,5,5,14^{*} 0\right)$ & 0.070376 & 0.024763 & 0.137682 \\
\hline & 20 & $(5,16 * 0,5,5,5)$ & 0.142366 & 0.110035 & 0.187844 \\
\hline & 20 & $\left(3^{*} 0,15,7^{*} 0,5,8^{*} 0\right)$ & 0.050102 & 0.031553 & 0.127175 \\
\hline \multicolumn{6}{|c|}{$\lambda=0.8$} \\
\hline$n$ & $m$ & Scheme & $\tilde{\sigma}_{1}$ & $\tilde{\sigma}_{2}$ & $\hat{\sigma}$ \\
\hline \multirow{7}{*}{10} & 10 & $\left(10^{*} 0\right)$ & 0.102902 & 0.070663 & 0.127828 \\
\hline & 6 & $\left(3^{*} 0,2,2,0\right)$ & 0.066990 & 0.051407 & 0.052529 \\
\hline & 6 & $(2 * 0,4,3 * 0)$ & 0.147022 & 0.108045 & 0.088532 \\
\hline & 6 & $(4,5 * 0)$ & 0.159449 & 0.112451 & 0.121885 \\
\hline & 6 & $\left(5^{*} 0,4\right)$ & 0.035599 & 0.036005 & 0.020392 \\
\hline & 5 & $(2 * 0,5,2 * 0)$ & 0.180094 & 0.108128 & 0.074795 \\
\hline & 5 & $(5,4 * 0)$ & 0.182012 & 0.132340 & 0.117633 \\
\hline \multirow{5}{*}{15} & 15 & $\left(15^{*} 0\right)$ & 0.071527 & 0.047072 & 0.128779 \\
\hline & 10 & $(5,9 * 0)$ & 0.105111 & 0.072178 & 0.124625 \\
\hline & 10 & $(4 * 0,3,3 * 0,2,0)$ & 0.051678 & 0.042403 & 0.074198 \\
\hline & 10 & $\left(0,3,6^{*} 0,2,0\right)$ & 0.048937 & 0.041798 & 0.098899 \\
\hline & 10 & $\left(2 * 0,1,0,2,0,2,3^{*} 0\right)$ & 0.084427 & 0.060247 & 0.069177 \\
\hline \multirow{5}{*}{20} & 20 & $\left(20^{*} 0\right)$ & 0.054423 & 0.034574 & 0.127387 \\
\hline & 15 & $\left(3^{*} 0,2,4^{*} 0,3,6^{*} 0\right)$ & 0.066006 & 0.044951 & 0.080022 \\
\hline & 10 & $\left(5,2^{*} 0,5,6^{*} 0\right)$ & 0.102578 & 0.068214 & 0.098566 \\
\hline & 10 & $\left(2^{*} 0,1,0,2,0,2,2^{*} 0,5\right)$ & 0.020416 & 0.021247 & 0.012089 \\
\hline & 10 & $\left(2^{*} 0,3,0,2,0,2,2^{*} 0,3\right)$ & 0.037316 & 0.041381 & 0.021111 \\
\hline \multirow{6}{*}{30} & 30 & $\left(30^{*} 0\right)$ & 0.037626 & 0.023119 & 0.127778 \\
\hline & 20 & $\left(3^{*} 0,5,3^{*} 0,5,12^{*} 0\right)$ & 0.053574 & 0.034622 & 0.099966 \\
\hline & 20 & $\left(2^{*} 0,10,17^{*} 0\right)$ & 0.051490 & 0.032389 & 0.124625 \\
\hline & 20 & $(9 * 0,10,10 * 0)$ & 0.055568 & 0.037093 & 0.076462 \\
\hline & 15 & $\left(5,6^{*} 0,10,7^{*} 0\right)$ & 0.070859 & 0.049199 & 0.073894 \\
\hline & 15 & $(10,6 * 0,5,7 * 0)$ & 0.068826 & 0.046658 & 0.078957 \\
\hline \multirow{4}{*}{40} & 20 & $\left(8^{*} 0,10,10,10^{*} 0\right)$ & 0.052078 & 0.034782 & 0.078689 \\
\hline & 20 & $\left(2^{*} 0,5,5,5,5,14^{*} 0\right)$ & 0.054582 & 0.035106 & 0.111600 \\
\hline & 20 & $(5,16 * 0,5,5,5)$ & 0.007270 & 0.007829 & 0.005961 \\
\hline & 20 & $\left(3^{*} 0,15,7^{*} 0,5,8^{*} 0\right)$ & 0.056001 & 0.037603 & 0.073094 \\
\hline
\end{tabular}

\section{References}

Balakrishnan, N. (1989). Approximate MLE of the scale parameter of the Rayleigh distribution with censoring, IEEE Transactions on Reliability, 38, 355-357.

Balakrishnan, N. and Aggarwala, R. (2000). Progressive Censoring: Theory, Methods and Applications, Birkhäser, Boston.

Balakrishnan, N. and Asgharzadeh, A. (2005). Inference for the scaled half-logistic distribution based on progressively Type-II censored samples, Communications in Statistics - Theory \& Methods, 34, 73-87. 
Balakrishnan, N. and Nevzovor, V. B. (2003). A Primer on Statistical Distributions, John Wiley \& Sons, New York.

Balakrishnan, N. and Wong, K. H. T. (1991). Approximate MLEs for the location and scale parameters of the half-logistic distribution with Type-II right-censoring, IEEE Transactions on Reliability, 40, 140-145.

Balakrishnan, N., Kannan, N., Lin, C. T. and Ng, H. K. T. (2003). Point and interval estimation for Gaussian distribution based on progressively Type-II censored samples, IEEE Transactions on Reliability, 52, 90-95.

Balakrishnan, N., Kannan, N., Lin, C. T. and Wu, S. J. S. (2004). Inference for the extreme value distribution under progressive Type-II censoring, Journal of Statistical Computation \& Simulation, 74, 25-45.

Balasooriya, U., Saw, S. L. C. and Gadag, V. (2000). Progressive censored reliability sampling plans for the Weibull distribution, Technometrics, 42, 160-167.

Han, J. T. and Kang, S. B. (2008). Estimation for the double Rayleigh distribution based on multiply Type-II censored samples, Communications of the Korean Statistical Society, 15, 367-378.

Johnson, D. (1997). The triangular distribution as a proxy for the beta distribution in risk analysis, The Statistician, 46, 387-398.

Kang, S. B. (1996). Approximate MLE for the scale parameter of the double exponential distribution based on Type-II censored samples, Journal of the Korean Mathematical Society, 33, 69-79.

Keefer, D. L. and Verdini, W. A. (1993). Better estimation of PERT activity time parameters, Management Science, 39, 1086-1091.

Kim, C. S. (2006). On estimating Burr Type-XII parameter based on general Type-II progressive censoring, The Korean Communications in Statistics, 13, 89-99.

Lin, C. T., Wu, S. J. S. and Balakrishnan, N. (2006). Inference for log-gamma distribution based on progressively censored data, Communications in Statistics-Theory and Methods, 35, 1271-1292.

Lee, H. J., Han, J. T. and Kang, S. B. (2008). Estimation for a triangular distribution based on multiply Type-II censored samples, Journal of the Korean Data \& Information Science Society, 19, 319-330.

Moder, J. J. and Rodgers, E. G. (1968). Judgment estimates of the moments of PERT type distributions, Management Science, 15, 76-83.

Ng, H. K. T., Chan, P. S. and Balakrishnan, N. (2002). Estimation of parameters from progressively censored data using EM algorithm, Computational Statistics \& Data Analysis, 39, 371-386.

Seo, E. H. and Kang, S. B. (2007). AMLEs for Rayleigh distribution based on progressive Type-II censored data, The Korean Communications in Statistics, 14, 329-344.

Viveros, R. and Balakrishnan, N. (1994). Interval estimation of parameters of life from progressively censored data, Technometrics, 36, 84-91.

von Drop, J. R. and Kotz, S. (2002). A novel extension of the triangular distribution and its parameter estimation, The Statistician, 51, 63-79.

[Received August 2008, Accepted September 2008] 